\title{
Espacios habitables sensibles: Microorganismos como herramientas de diseño.
}

Sensitive habitable spaces: Microorganisms as design tools.

\author{
Fernando Meneses-Carlos \\ Universidad de Monterrey, México \\ fernando.meneses@udem.edu \\ Daniela Frogheri \\ Universidad de Monterrey, México \\ daniela.frogheri@udem.edu
}

\begin{abstract}
This article aims to validate the possibility of including technology from micobiologies and synthetic biology in architecture and design. For this analysis, five projects are presented: a project of our own, developed by the research group, another with a direct application in architecture and three additional projects form the world of microbiology, which review topics such as energy generation, materials production and improving air quality thought microorganisms. This analysis, aims to legitimate, and expose the advantages and limits of a potential union between the molecular world and the design of the habitable space.
\end{abstract}

Keywords: Architecture, Microorganisms, Sensitive, Emergency; Monads

\section{Introducción}

En el presente artículo se muestra una recopilación de intervenciones de programación de microorganismos con posible aplicación en la arquitectura y el diseño. Para ponernos en contexto, partiremos con la idea de Stéphane Le Duc expuesta en 1910, basada en la posibilidad de una biología que no sólo fuera producto de la contemplación de la naturaleza, sino que surgiera del estudio puro de los sistemas abstractos sin la contemplación de lo existente en la vida natural, con esto se crearía una nueva área del conocimiento que ahora conocemos como biología sintética. (Le Duc, 1910)

La biología sintética ahora es una nueva área de la biología molecular, y desde Le Duc hasta ahora ha permitido que un gran número de investigadores trabajen en el estudio de los sistemas biológicos desde la abstracción, llegando a proponerse temas tan profundos como el chasis genómico mínimo, donde se busca encontrar el modelo mínimo biológico para generar vida.

En otra área del conocimiento, pero en la misma dirección encontramos las mónadas (Leibniz, 1981) donde se describe una metafísica basada en elementos infinitesimales definidos como la unidad mínima de la materia, lo relevante aquí, es que las mónadas son entendidas como elementos sensibles, lo que permite pensar en una realidad generada a partir de las múltiples combinaciones de las partes, pero sobre todo y con especial atención en lo sensible de cada una.

En este contexto sería válido preguntarnos si este tipo de tecnología se puede aplicar en el diseño del espacio habitable; existen precedentes como el Proyecto de Barcelona Genética en el 2000 (Estévez, 2004) donde se proponía manipular genéticamente un limonero con la proteína GFP para iluminar Barcelona, también existen algunas intervenciones artísticas donde se modifican animales para efectos plásticos, pero la pregunta entonces es si hoy, cien años después del invento de la biología sintética y a diecisiete de la primera aplicación de la genética en la arquitectura, será válido pensar en aplicaciones tangibles de estas tecnologías en beneficio del espacio habitable.

El presente artículo intenta recabar algunos de los ejemplos más representativos de las tecnologías de la biología sintética que se podrían incluir en el campo de la arquitectura y el diseño, exponiendo los principios básicos de la manipulación genética, así como algunas exploraciones conceptuales de posibles aplicaciones. En el fondo, la hipótesis general sería que de ser posible la programación de microorganismos como herramienta de diseño, sería más factible pensar en espacios resilientes más allá de su propio diseño o construcción. Sería posible pensar espacios cuasi vivos que funcionen como sensores y actuadores biológicos en la vida cotidiana

De esta manera se podría pensar en la posibilidad de diseñar un espacio habitable mediante la programación de microorganismos, así en un futuro muchos problemas de las ciudades se podrían abordar a nivel molecular, y generar alternativas de mejoras como menor consumo energético, manejo de residuos e incluso aumentando la resiliencia de las ciudades en general.

En este contexto, el artículo no es una aportación en materia de avances de la microbiología, es solamente un análisis de la posibilidad de incluir la microbiología en el diseño, en este sentido, se analizará un ejemplo desarrollado dentro de nuestro grupo de investigación y se analizaran ejemplos realizado en este marco conceptual, intentando analizar la posibilidad, retos y límites de la aplicación de la microbiología en el diseño. 


\section{Metodología}

En este proyecto se unieron diferentes grupos académicos y de investigación, tales como el FabLab Monterrey, Biohackademy, la Maestría de Arquitecturas Avanzadas y el equipo de Microbiología de la Universidad de Monterrey. El objetivo principal era lograr que un grupo de arquitectos, diseñadores y artistas lograran modificar genéticamente a unas bacterias, con el fin de analizar la posibilidad de la implantación de estas tecnologías en el diseño y la creación.

El artículo pretende exponer los temas tratados en el taller hacking bacterias, y en este marco, se buscarán analizar proyectos que ya se están desarrollando hoy dentro de la biología sintética, y que ya podrían ser aplicados en temas de diseño arquitectónico, arte y el diseño en general. Para iniciar y a manera de premisas se analizarán los conceptos de biología sintética y de las mónadas, con la intención de poner en la mesa los temas que posteriormente nos permitirán establecer el debate final.

En el taller hacking bacterias se amaizará a detalle el proceso de hackeo biológico de una bacteria, a partir de conceptos como el DNA y RNA. El hackeo trata de la manipulación de las secuencias genéticas, para esto es necesario leer, interpretar, traducir y reprogramar las secuencias, el fin último de este proceso es la generación de proteínas, que en el fondo son el motor del mundo molecular.

Ya con estos conceptos y procesos en la mente, y recordando que somos diseñadores y no genetistas, se analizarán proyectos como el de Barcelona genética, como un ejemplo real aplicado al diseño, y tres proyectos más, donde desde los microorganismos se están generando materiales, generando energía y mejorando la calidad del aire. Los proyectos se analizarán con la intención de reunir referencias de posibles aplicaciones reales, con la intención de abrir la puerta a nuevas ideas de aplicación.

Finalmente se busca validar la posibilidad de la inclusión de las tecnologías de biología molecular, no solamente como tecnologías existentes que se pueden elegir de un menú, sino que estas tecnologías sean parte de las posibilidades del proceso creativo del diseño, y por tanto, surjan retos en materia y a nivel molecular desde el diseño.

\section{Ideas previas}

Antes de entrar en materia sería conveniente poner sobre la mesa dos ideas, la primera es el cambio intelectual que implico la biología sintética en el mundo de las bioingenierías, y la segunda, las mónadas de Leibniz, una idea que desde la filosofía nos ofrece una aproximación profunda y detallada de la idea de apostar por las unidades mínimas, como lo hace la biología molecular, es decir, usaremos la filosofía de Leibniz para validar la realidad molecular en el mundo o escala física en la que sucede el diseño.

\section{Biología sintética}

La manipulación genética es una de las grandes revoluciones de nuestros tiempos, pero es importante resaltar un cambio intelectual en esta área del conocimiento con la llegada de la postura sintética, donde el postulado es sencillo y radical, la idea principal es que la tradición biológica solamente se limita a observar la vida, en el caso de la genética sigue observando la vida, sólo que a nivel molecular pero en el fondo sigue siendo todo a partir de la decodificación de los seres existentes. En lugar de esto la biología sintética expone otra posibilidad, planteando la opción de generar vida, pero sin replicar algo de la naturaleza, es decir, si ya se tiene un código y unas reglas, entonces es posible explorar la combinación de éstas solamente desde sus combinaciones racionales y lógicas, es decir, generar seres que no sean la copia de algo ya existente.

Este cambio de paradigma abre las puertas a todo un universo de posibilidades, y también es cierto que este es un tema que tiene que estar acompañado de una reflexión sobre los problemas éticos, pero el potencial reside en la posibilidad de programar organismos con funciones particulares. En la actualidad existen proyectos muy robustos como el proyecto CRISPR Clustered Regularly Interspaced Short Palindromic Repeats (Jansen, Embden, Gaastra, \& Schouls, 2002), donde ya es posible la edición genética, o proyecto BioBricks ("BioBricks," 2017) donde a manera de repositorio de material bilógico bajo el proyecto Open Material The BioBrick Public Agreement (BPA) y el Transfer Agreement (OpenMTA) el cual contempla un protocolo de transferencias de material bilógico, y The National Center for Biotechnology Information NCBI ("NCBI," 2017), con lo que en definitiva se ofrece toda una gama de herramientas para la ingeniería biológica que parece y promete ser el futuro del bio-diseño.

También existen redes como el International Genetically Engineered Machine "iGEM" ("iGEM," 2017), la que sin duda es una de las redes más importantes de expertos en biología sintética. Con todo esto, podemos decir que hoy ya es posible programar microorganismo con una función específica, lo que pone sobre la mesa del diseño, la pregunta sobre cómo, dónde y para qué necesitaría el diseño las bio-tecnologías.

\section{Mónadas}

La postura filosófica de Leibniz fue publicada por primera ocasión en 1714 (Leibniz, 1981), y abordarla ahora implica entender que esta idea se presentó en un momento donde la comunidad intelectual aún no aceptaba la idea de átomo, así que hoy en día gran parte de esta postura la podríamos ver como obvia, ya que actualmente sería difícil que alguien no entienda la idea de átomo, pero incluso podríamos decir que más allá de lo obvio, la misma microbiología y muchas otras áreas son hijas de las ideas como el átomo y sin duda del planteamiento filosófico de las mónadas.

En este sentido, podríamos decir que para Leibniz en la realidad no hay discontinuidad, es decir, la realidad es infinitesimal, es imposible conocerla en totalidad, lo que existen para nosotros son solamente diferentes interpretaciones, pero esta idea también la planteaba en el sentido de la escala de las cosas, para Leibniz el átomo era obvio, pero también defendía que no existe un corte de realidad entre el mundo atómico y el mundo de las cosas, o 
incluso el mundo a la escala del universo. Así que Leibniz estaría de acuerdo con la biología molecular, pero puede ser que preguntaría y porque la separamos como si tratara de otra realidad, si lo ralamente importante está en entender la continuidad de la realidad.

Otra idea importante es la de las verdades de la razón y las verdades de los hechos, para Leibniz no hay una realidad única, la verdad de los hechos es todo el conocimiento que es necesario validar en el mundo físico, es todo lo que necesita comprobación, estas verdades son primitivas. Por el otro lado están las verdades de razón, estas son verdades sofisticadas, y surgen de la lógica pura, no de la realidad y por tanto no requieren comprobación.

Si lo pensamos un poco, el cambio intelectual de la biología a la biología sintética, es en esencia un cambio de la verdad de hechos a la verdad de razón. Y por esto, según la filosofía de Leibniz, la biología sintética en la categoría de las verdades de razón, llega más a lo profundo de la realidad, no solamente porque su escala es pequeña, sino porque es una verdad sin necesidad del hecho.

Para entender la verdad de razón de Leibniz, es necesario hablar de otros tres conceptos, el primero es la del sujeto y el predicado, por ejemplo, en la frase el pez nada, el predicado nadar está implícito en el sujeto pez, de esta manera no es necesario ir hasta donde esté un pez para dar por cierto que el pez nada, dado que la acción está implícita en el sujeto.

El segundo concepto es la permanencia, en este sentido la evaluación verifica que el sujeto a pesar de que cambie su predicado el sujeto se mantenga, por ejemplo, el pez come, el pez juega, en estas frases el sujeto siempre es el mismo y no pierde su esencia. Por último, la inmutabilidad en el sentido que un sujeto que es esencial no puede ser sustituido por otro sujeto y no puede ser predicado de otra cosa, así tendríamos que el nado es pez, el niño nada son verdades que no pueden ser validadas desde la razón, para demostrarlas tendríamos que comprobarlas en la realidad y en el contexto en el que se estén expresando.

Dicho lo anterior, la mónada es la esencia del sujeto, es la parte más pequeña, esa parte que es imposible dividir, es la unidad mínima, Leibniz pensaba que el átomo era real, pero sabía que el átomo también está compuesto por otras partes, y estas partes, por otras partes aún más pequeñas hasta una dimensión infinitesimal, en ese punto es donde se encontraban las mónadas.

Las mónadas son la parte sensible de la realidad, esta parte es muy importante, porque, si la teoría de Leibniz es correcta, entonces para las áreas del diseño, que están muy interesadas en la percepción y las emociones, la mónada es el equivalente al mundo molecular de la biología, y en este sentido merecería la pena plantear la posibilidad de una decodificación de lo sensible y por tanto una sensibilidad sintética, que exploré los elementos básicos de lo sensible.

\section{Análisis de proyectos}

Ahora analizaremos cinco proyectos, el primero es el taller hacking bacterias realizado por nuestro grupo de investigación y donde el objetivo fue entender las bases teóricas y prácticas de la reprogramación genéticamente una bacteria, aquí se explicará paso a paso el proceso de, con la intención de poner sobre la mesa el fondo práctico del tema.

El segundo es el proyecto de Barcelona Genética, que sería el primero donde se incluye de manera real la genética en la arquitectura, el tercero el Geobacter Project, donde a partir de la Geobacter Metallireducens se genera energía, el cuarto es el MycoWorks, donde se desarrollan materias por medio de microorganismo y el quinto proyecto es el de BetterAir, donde por medio de microbiología se mejora la calidad del aire.

Con estos proyectos se espera tener información tangible y suficiente como para validar la posibilidad de la unión continua del mundo microbiológico con el del diseño habitable. Así como los retos, límites y analizando la fundamentación teórica de las relaciones entre los dos mundos.

\section{Hacking Bacterias}

El primer proyecto que analizaremos es el hacking bacterias, fue un curso para los alumnos de la Maestría en Arquitecturas Avanzadas MAA y los profesores del departamento de arquitectura de la Universidad de Monterrey, el primer punto de trabajo fue explicar a los arquitectos, diseñadores y artistas cómo funciona el mundo de los microrganismos y la manipulación genética, esta parte estuvo a cargo del Dr. Ernesto Ladrón de Guevara y el Dr. Jorge A. Marcos Víquez de Biohackademy.

El taller fue planteado para que un diseñador entendiera los conceptos clave de la manipulación genética, sea en la parte teórica como en la práctica, la idea es que un diseñador experimente y mida lo posible y factible de incluir estas tecnologías en su proceso de diseño. En taller los alumnos lograron tener su primer contacto con el mundo molecular, y en especial con las bacterias. (Figura 1).

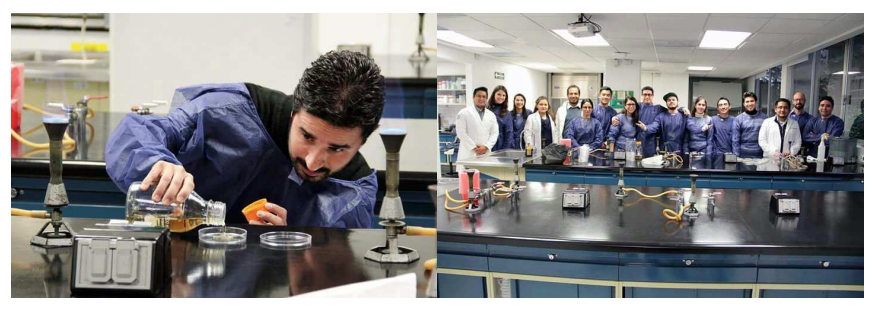

Figura 1: Taller hacking bacterias, alumnos y profesores de la MAA.

El taller inicio presentando los conceptos de DNA y RNA: ácido desoxirribonucleico y ácido ribonucleico, respectivamente por sus siglas en inglés, porque que son la base natural para la transmisión de la información genética, son moléculas muy sencillas, las dos están formados por cadenas de monómeros que a manera de nucleótidos se enlazan uno detrás del otro por enlaces fosfodiéster, y cada nucleótido está formado por tres elementos: (i) monosacárido de cinco carbonos, pentosa y del tipo ribosa, (ii) fosfato y (iii) 
una base nitrogenada, que es donde está lo interesante, ya que solamente existen cuatro bases nitrogenadas para cada uno, para el DNA tenemos (T) Timina, (C) Citosina, (A) Adenina y $(G)$ Guanina, y para el RNA tenemos (U) Uracilo, (C) Citosina, (A) Adenina y (G) Guanina. (Tortora, Funke, \& Case, 2007).

Esto es muy importante porque reducir el alfabeto del AND y RNA en TCAG y UCAG respectivamente, es equivalente a cuando la informática llego al 0 y 1 (Model, 2009), es decir, bajo este modelo es posible codificar y decodificar la secuencia de estas cadenas, y con esto, la secuencia genética. Otro detalle importante es que el DNA es una hebra bicatenaria y el RNA es monocatenaria, en palabras simples y si pensamos en la imagen del DNA cuando se representa como una escalera, esto sería bicatenaria, que tiene las dos hebras, mientras que el RNA es monocatenaria, es como si cortáramos la escalera a la mitad y se quedara solamente con un soporte vertical, con una sola hebra.

Si lo anterior es claro, entonces podemos pasar a la transcripción, donde lo primero es decir que existen dos tipos de células, las eucariotas y las procariotas, en este caso se hablará de las procariotas, que son las que tienen las bacterias.

El proceso de transcripción inicia con las enzimas helicasas, las cuales tienen la función de separar las dos hebras del DNA convirtiéndolo en RNA, aquí entran al juego las proteínas estabilizadoras que impiden que las dos hebras se unan de nuevo, y aquí entra la enzima RNA polimerasa que se encarga de hacer una réplica del RNA, al que se llamara RNA mensajero, este RNA pasa el ribosoma para sintetizar la proteína.

Para las células las eucariotas, es decir las que tienen un núcleo, en este caso además de lo anterior es necesario hacer un proceso de corte y empalme, el cual consiste en eliminar el intrón, que es la parte del RNA que no es útil para duplicar el DNA y que en general está en el centro del RNA, después de esto sólo nos quedaran los exones, ahora es momento de incluir el CAP y el poli-A, que son los que hacen de inicio y fin de la secuencia, el CAP ayuda para entrar al ribosoma y el poli-A para encapsularlo e impedir que se peguen más secuencias, y ahora si pasa al ribosoma y sintetiza la proteína (Pena, 1988).

Ahora nos queda la última parte de traducción, donde se identificarán las secuencias del RNA mensajero y se dosificarán según las secuencias, agrupadas en unidades de tres bases a las que se les llamara codón, sobre estos paquetes de tres, se pegarán otras tres bases que se denominan el anti-codón, con esto se generaran los aminoácidos Phenylalanine, Leucine, Isoleucine, Valine, Serine, Proline, Threonine, Alanine, Tyrosine, Histdine, Glutamine, Asparagine, Lysine, Aspartic, Cysteine, Tryptopha, Arginine, Serine, Glycine, Methionine como el start y el stop. Tal como lo podemos ver en la (Tabla 1).
Tabla 1: Secuencia de aminoácidos en la cadena codificada del RNA mensajero (Rosenberg \& Rosenberg, 2012, p. 103).

\begin{tabular}{|c|c|c|c|c|c|c|c|c|c|}
\hline & t & & & & & & & & \\
\hline & UUU & Phe & UCU & Ser & UAU & Tyr & UGU & Cys & $\mathbf{U}$ \\
\hline & UUC & Phe & UCC & Ser & UAC & Tyr & UGC & Cys & C \\
\hline & UUA & Leu & UCA & Ser & UAA & Stop & UGA & Stop & $\mathbf{A}$ \\
\hline & UUG & Leu & UCG & Ser & UAG & Stop & UGG & Tyr & $\mathbf{G}$ \\
\hline & CUU & Leu & $\mathrm{CCU}$ & Pro & $\mathrm{CAU}$ & His & CGU & Arg & $\mathbf{U}$ \\
\hline & CUC & Leu & CCC & Pro & CAC & His & CGC & Arg & C \\
\hline & CUA & Leu & CCA & Pro & CAA & GIn & CGA & Arg & $\mathbf{A}$ \\
\hline & CUG & Leu & CCG & Pro & CAG & Gln & CGG & Arg & $\mathbf{G}$ \\
\hline & AUU & Ile & ACU & Thr & AAU & Asn & AGU & Ser & $\mathbf{U}$ \\
\hline & AUC & $\mathrm{Ile}$ & ACC & $\mathrm{Thr}$ & AAC & Asn & AGC & Ser & C \\
\hline$A$ & AUA & IIe & ACA & $\mathrm{Thr}$ & AAA & Lys & AGA & $\operatorname{Arg}$ & $\mathbf{A}$ \\
\hline & AUG & Met & ACG & $\mathrm{Thr}$ & AAG & "Lys & AGG & $\mathrm{Arg}$ & $\mathbf{G}$ \\
\hline & GUU & $\mathrm{Val}$ & $\mathrm{GCU}$ & Ala & GAU & Asp & GGU & Gly & $\mathbf{U}$ \\
\hline & "GUC' & $\mathrm{Val}$ & GCC & Ala & GAC & Asp & GGC & Gly & C \\
\hline & 'GUA' & $\mathrm{Val}$ & GCA & Ala & GAA & "'mlu & GGA & Gly & $\bar{A}$ \\
\hline & GUG & Val & GCG & Ala & GAG & Glu & GGG & Gly & $\mathbf{G}$ \\
\hline
\end{tabular}

Ya que tenemos generadas los aminoácidos pasamos al RNA ribosomal, donde se ensamblan todos los aminoácidos para generar las proteínas. Con esto tendríamos completado el proceso de transformación de DNA a RNA para generar proteínas, de esta manera podemos concluir que es posible transferir la secuencia genética de un organismo desde su DNA a RNA, pero también podemos transferir de RNA a DNA, en el fondo es aquí donde sucede el hackeo biológico (PhD, Bernstein, Ingram, \& Hart, 2015). Así que podemos decir que las posibilidades de hackeo están de:

$$
D N A \rightleftharpoons R N A \rightarrow \text { Proteína }
$$

Donde el fin último es generar proteínas, que como sabemos es la materia que da vida al mundo molecular. En este caso el taller consistió en cultivar bacterias recolectadas en diferentes ambientes de la Universidad, esta parte del taller fue muy ilustrativa sobre el universo de bacterias con las que convivimos todos los días, y también para ver qué tan contaminados estas los ambientes que en ocasiones pensamos que están limpios. La segunda parte del taller consistió en hacker a una bastaría para hacerla fluorescente con la proteína sintética, siguiendo el procedimiento descrito anteriormente. (Figura 2)

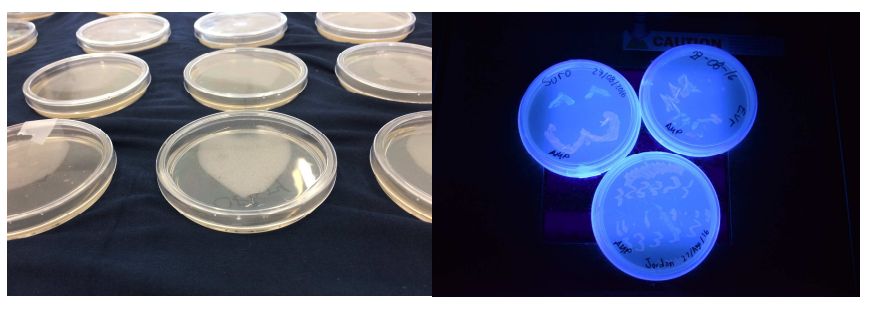

Figura 2: izq. Cultivo de bacterias en la UDEM, der. Bacteria hackeda con la proteína sintética por Biohackademy.

\section{Barcelona Genética: Arquitectura}

El segundo proyecto tiene sus inicios en la visión de evolución histórica de la arquitectura por parte de Alberto T. Estévez, y que se consolidaría con la apertura de una investigación formal y la apertura de un posgrado en Arquitecturas Genéticas en 2000, teniendo su culminación con la 
presentación del proyecto con el alcalde de Barcelona en el 2005. En la página oficial del grupo de investigación se describen tres fases, a saber, fase 1 de 2003 a 2005, con la creación genética de plantas bioluminiscentes para uso urbano y doméstico; fase 2 de 2007 a 2010, donde se desarrolló una bio-lámpara ahora con bacterias; fase 3 de 2011 a 2014, donde se desarrolló la idea de plantas con fin ornamental y dotadas de bacterias e insectos bioluminiscentes. (Estévez, 2017). Primera fase (Figura 3).

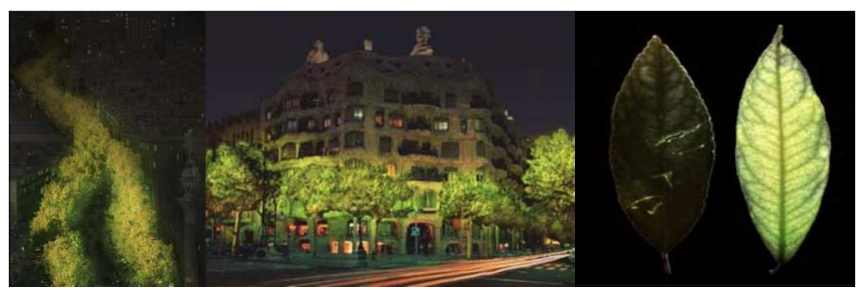

Figura 3: Barcelona Genética, fase 1. (Estévez, 2007)

Este proyecto es interesante no solamente por la innovación de la aplicación de la manipulación genética, ya que, en términos teóricos, la primera fase intentó modificar un limonero con la proteína GPF, pero desde el punto de vista de la manipulación genética, un limonero es organismo extremadamente complejo, por esto que sea un gran avance que en la fase 2 (Figura 4), se buscara modificar un organismo más sencillo como las bacterias, aquí podemos observar la bio-lámpara.
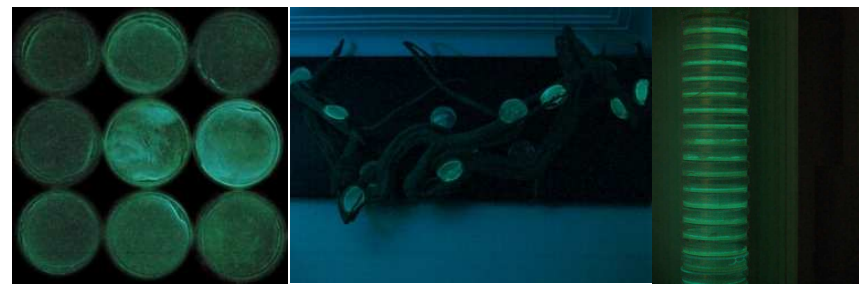

Figura 4: Barcelona Genética, fase 2. (Estévez, 2017)

El posible inconveniente de solamente trabajar con bacterias es que tienen un periodo de vida muy corto, por esto la idea de tener una base como una planta y que a partir de esta crezcan colonias de bacterias es un buen avance en el proyecto, tal y como se muestra en las imágenes de la fase tres de Barcelona Genética (figura 5).

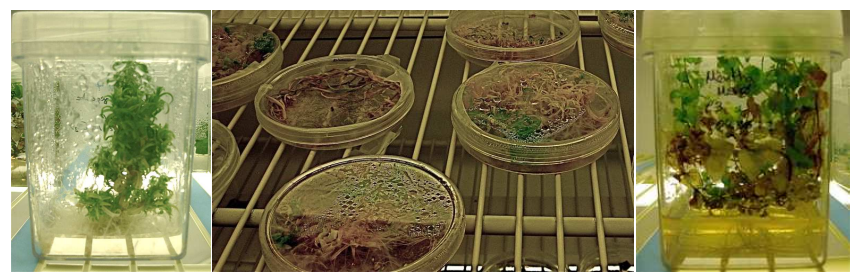

Figura 5: Barcelona Genética, fase 3. (Estévez, 2017)

En este sentido, el proyecto es un buen ejemplo de la aplicación de la genética en la arquitectura, y es de un investigación seria y pionera en el tema. En este proyecto se utilizó la GFP Green Fluorescent Protein. Ahora si recordamos un poco lo del taller de hacking bacterias, y si usamos el
BioBrick Public Agreement (BPA) o el National Center for Biotechnology Information NCBI, nos daremos cuenta que hoy tenemos a nuestro alcance, la secuencia genética de la GPF (Tabla 2), en este caso vemos la versión sintética EiraCFP_58 de Genes sintéticos para GFP no naturales.

Tabla 2: Secuencia de genes sintéticos para GFP no natural. ("GFPBioBricks," 2017)

ATGTCGTCTGGTACCAAATTGTTTGAAAAGGAAATCCCGTATATCACTGAACT
GGAGGGCGACGTCAATGGTATGAAGTTTACCATTCATGGTAAAGGTACCGG
CGATGCGAGCACGGGTACCATTAAAGCGAATTCATCTGCACTACGGGCGA
CGTTCCGGTCCCGTGGGCAACCTGGTGAGCACCCTGAGCTACGGTGTTCA
GTGTTTCGCCAAGTACCCGCCGCACATCAAGGATTTCTTTAAGAGCGCCATG
CCGGAAGGTTATGTTCAAGAGCGTACCATCACCTTCGAAGGCGACGGCGTG
TTTAAGACGCGTGCTGAGGTTACCTTTGAAACGGTTCTGTCTACAATCGTG
TCACGCTGAACGGCCAGGGTTTTAAGAAAGACGGTCACATTCTGCGTAAGA
ACGTTGCATTCCAATGCCCGCCAGATATTGTGTATATTTTCCTGACACCGT
TAACAATGGCATCCGCGTTGAGTCAACCAGGCGTACGATATTGAAGGTT
GACCGAAAACTGGTTACCAAATGCAGCCAAATGAATCGTCCGTTGGCGGG
CTCCGCGGCAGTGCATATCCCGCGTTATCATCACCTGAGCAAACACACCAA
ACTGAGCAAAGACCGCGACGAGCGCCGTGATCACATGTGTCTGGTAGAGGT
CGTGAAAGCGGTTGATCTGGACACGTATCAGTAA

Como comentamos en el mismo taller, hoy también es posible acceder al material biológico, con lo que hoy no tendríamos ningún problema para sintetizar la proteína GFP.

\section{Geobacter Project: Generación de energía}

El tercer proyecto es el Geobacter metallireducens fue descubierto 1987, y desde entonces es una de las bacterias más estudiadas, tanto que se generó el Geobacter Project, donde se comparten los descubrimientos y nuevas aplicaciones del Geobacter, También funciona como una comunidad de investigadores de todo el mundo que trabajan en conjunto, el Geobacter tiene propiedades que lo hacen ideal para la biorremediación y para la generación de energía.

Lo especial de esta bacteria es que oxida compuestos orgánicos y algunos metales, de tal manera que es capaz de generar electrones a partir de los iones, pero sin residuos, esta bacteria tiene un metabolismo que pone en el equilibrio la oxidación, algo parecido a lo que hacen las plantas con el bióxido de carbono.En las siguientes imágenes podemos ver de izquierda a derecha, una foto del Geobacter metallireducens y una representación en 3D a nivel molecular. (Figura 6)

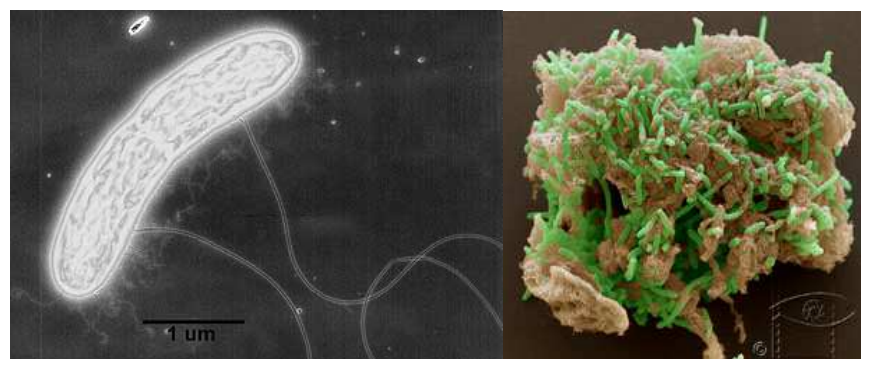

Figura 6: Geobacter metallireducens (“Geobacter," 2017)

Sin duda es una bacteria que será muy popular en el futuro, hoy en día tenemos acceso a la secuencia genética completa de la versión GS-15 (Tabla 3). Aquí podemos notar que la secuencia genética del GFP es muy pequeña en comparación 
de la secuencia del GS-15, en este artículo solo se incluyeron las primeras líneas, pero en el link se puede acceder a la secuencia completa, para dimensionar la diferencia e imaginar lo que implicaría manipular organismos más complejos.

Tabla 3: Secuencia del Geobacter Metallireducens GS-15. (Aklujkar et al., 2009)

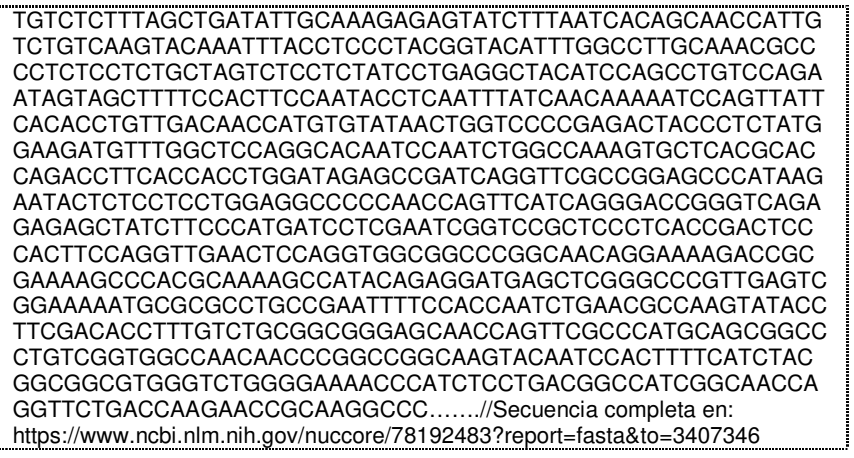

\section{MycoWorks: Producción de materiales}

El cuarto proyecto es un buen ejemplo de cómo los microrganismos pueden ser productores de materiales con cualidades específicas, es decir, como diseñar y generar materiales desde los microrganismos, este proyecto trabaja a partir del mycelium ("MycoWorks," 2017), una fibra trabaja como chasis, sobre esta puedes crecer hongos y bacterias.

El mycelium tiene una forma de crecimiento diferente, lo que permite que el tamaño de sus colonias sea considerablemente grande, y lo que la convierte en ideal para el desarrollo de materia a nuestra escala, por decirlo de alguna manera.

El mycelium es utilizado también en producción agrícola, como el cultivo de champiñones, pero en MycoWorks lo utilizan para fabricar piel sintética, logrando con esto piel sin el sacrificio de animales, pero también desarrollan materiales estructurales, como podemos ver en las siguientes imágenes (Figura 7).
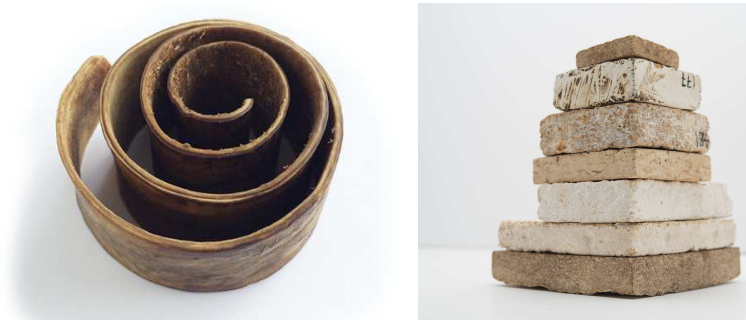

Figura 7: Materiales generados por microrganismos, piel y material estructural. ("MycoWorks," 2017)

Este ya sería un excelente ejemplo de la posibilidad del mundo molecular en el mundo escala persona, otras ventajas que tiene fabricar material de esta manera es que es muy amigable con el medioambiente y gasto de energía es renovable, ya que se utiliza el propio metabolismo del microrganismo para generar el material.

El mycelium es homokaryotic, es decir que en su reproducción la célula no es por la unión de dos, ya que las células son idénticas, así que su reproducción consiste en generar células idénticas, y no de combinación que suele ser lo habitual.

Aquí lo relevante es que no solamente podemos cambiar la materia a partir de la producción de proteínas, este es un buen ejemplo de que también se puede hacer usando las cualidades del metabolismo delos microorganismos. En la siguiente tabla tenemos la secuencia genética de la proteína del Mycelium aéreo (Tabla 4).

Tabla 4: Secuencia de la formación de la proteína del Mycelium Aéreo SCO4184. (Huang et al., 2013)

ATGAGCACACCGAGTACGGGGCGGTCGTTCGGGACTGCCGCGTTGACATG TCCGAGTGGCCGGGGCGGTCCGAGCGGTCCGGCCGGCCTGGGTGGTCTG GAGGCACCCCGGGCGCCCGTGCAGCGCACGGACAGCCCCGAGCTGCCGG CGGATGCCCCGCAGGGGGACCTGACCGCGCTGAGCCTGCCGGAGCTGCG CACGCTGCGCCGGGACGCCCAGCGCGAGGAGGCCGACCTCAGTTACGTAC GGCGGCTGCTCCAGGGCCGTATCGACATCCTGCGCGCGGAGCTGGCGGG GCGCGGGCCGGCGACGCCGTCGTCGTCCGTGGTCACCACCGCGGCGACC GGGTCGGTGGTGGAGCGGCTCTCGGAGATCCTCGCCGACGCGCCGGCCC GGCAGCGCTCCTCCGCCCGGCACGTGACGCTGGCCACCCCGCGCAGCGA GGAGTGCCGGCGGCTGGCCGCGGAGATGATGGGGGAGGTGGAGCTGTCC GACCTGACGGCCCGCACCGACGTCGAGCTGACCGCCGGGATGGGGCGGC TCGTCCGCTACGAGCAGCAGGTCTCCCGGAGCCGGCAGCGGCTCCAGCGC ACGGCGGACGGCTGCAGCGCGGAGATCGCGCGCCGGTACCGTGAGGGGG AAGCACAAGTCGACGACCTGCTCGTGTGA

\section{BetterAir: Mejora de la calidad del aire}

El quinto proyecto trata sobre la mejora de la calidad del aire, la teoría nos dice que existen bacterias que son peligrosas para el ser humano, pero también hay bacterias que son muy positivas, y como es de imaginarse, las bacterias buenas neutralizan a las bacterias malas $y$ a todos los microorganismos que están en el ambiente.

Por esto cuando usamos antibacteriales, lo que sucede es que matamos a todas las bacterias, a las malas, pero también a las buenas, y esto nos deja desprotegidos de todos los otros microorganismos que están en el ambiente, como los ácaros (Figura 8).

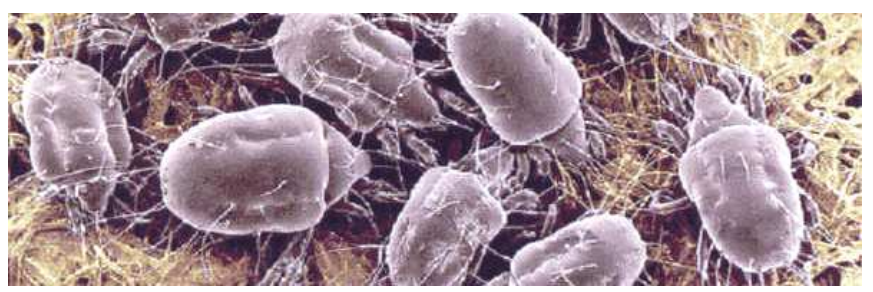

Figura 8: Ácaros del polvo al microscopio. (“USDA,” 2017)

Para solucionar esto, el equipo de BetterAir, utilizó probióticos en este caso usaron la bacteria Bacillus Ferment, la técnica es muy sencilla, se lanzan los probióticos al aire, y estos se encargan de consumir los restos excremento de ácaros, polen, células muertas. El proceso de purificación de aire es a nivel molecular, logrando con esto una intervención en el espacio habitable.

En la siguiente imagen podemos ver una bacteria Bacillus subtilis (Figura 9). En este caso el Bacillus se utilizó como tratamiento para curar una planta que estaba infectada por patógenos, como se puede ver en las imágenes, el Bacillus 
mejoró notablemente a la planta. Algo parecido es lo que el equipo de BetterAir hace con los probióticos en el aire.
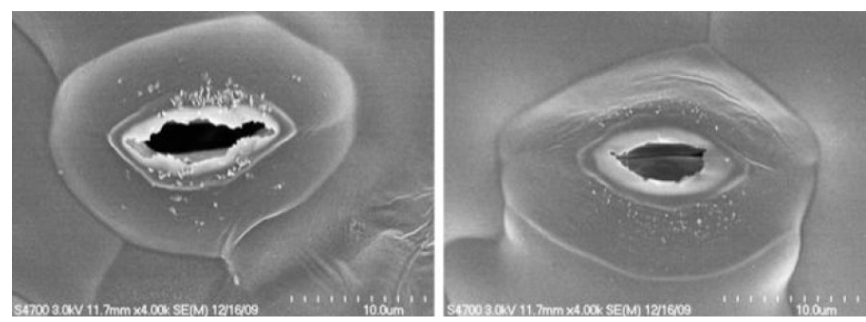

Figura 9: izq. una planta con patógenos, der. la misma planta con el Bacillus subtilis como tratamiento (Kumar et al., 2012)

Este ejemplo nos invita a penar que algunos problemas de contaminación se podrían solucionar desde el mundo de los microorganismos, y también es una oportunidad para ver que otra cualidad que podríamos usar de los microorganismos, es la relación que existe entre ellos.

Podríamos, por ejemplo, pensar en solucionar los problemas de contaminación del aire den las grandes ciudades, así como mejorar las calidades del aire en espacios cerrados, e incluso podríamos poner en comunicación los microorganismos de espacios cerrados con espacios abiertos en la ciudad. A continuación, la secuencia genética de Bacillus (Tabla 5).

Tabla 5: Secuencia del Macillus subtilis Pta1060. (Meijer, 2014)

TTGATTTATTCATCTGAAAATGATTATAGCATCCTCGAAGATAAAACCGCAAC
AGGTAAAAAGCGGGATTGGAAGGGGAAAAAAGACGGACGAACCTCATGG
CGGAGCATTACGAAGCGTTAGAGAGTAAGATTGGGGCACCTTACTATGGCA
AAAAGGCTGAAAACTAATTAGTTGTGCAGAGTATCTTTCGTTTAAGAGAGA
CCCGGAGACGGGCAAGTTAAAACTGTATCAAGCCCATTTTGTAAAGTGAGG
TTATGTCCGATGTGTGCGTGGCGCAGGTCGTTAAAATTGCTTATCACAATA
AGTTGATCGTAGAGGAACCAATAGACAGTACGGCTGCGGATGGATTTTCT
CACGCTGACGATTCGAAATGAAAGGGAGAACGGCTGAAGCACAAATTC
TGCGATGATGGAAGGCTTAGGAAACTGTTCCAGTACAAAAAATAAAAACT
TCGGTTCTTGGATTTTTCAGAGCTTTAGAGATTACCAAAAATCATGAAGAAGA
TACATATCATCCTCATTTTCATGTGTTGATACCAGTAAGGAAAATTATTTTG
GGAAAAACTATATTAAGCAGGCGGAGTGGAC......//Secuencia completa en:
https://www.ncbi.nIm.nih.gov/nuccore/NC 001766.1.

\section{Discusión}

Estos proyectos son solamente una muestra, de lo que se puede hacer desde el mundo de los microorganismos, pero con este repaso rápido análisis, nos podemos dar cuenta, que en la actualidad estas tecnologías ya están resolviendo algunos problemas de la vida cotidiana, la pregunta entonces es sí la arquitectura y el diseño tienen que ser parte de esta historia.

Un primer debate es si estas tecnologías solamente se reducen a generar nuevos materiales o cambiar cosas en lo micro, pero esto sería un error de dimensión según la postura de Leibniz, es claro que, si no se entiende la naturaleza de un ladrillo, sería muy complicado que un arquitecto diseñara un edificio, y ahora tenemos la oportunidad de entender los ladrillos que generan el ladrillo.

Otro tema importante, y retomando la propia postura de Leibniz sobre las verdades de la razón y las verdades de los hechos, es que en el caso de la arquitectura no solamente estamos en la verdad de la razón, entendiendo con esto todas las ideas sofisticadas de la lógica, la arquitectura también existe en la verdad de los hechos, es decir en las ideas que tienen que ser comprobadas en el mundo físico.

Con esto, podríamos decir que la biología sintética podría solucionar los problemas de la razón y no necesariamente los de los hechos, o en otras palabras, que la solución arquitectónica tiene sus propias implicaciones en la escala en la que existe, y por tanto, no se debería esperar que la escala molecular la solucione por completo.

Pero esto no es negativo, por el contrario, nos define un límite de acción, la pregunta entonces se torna más interesante, ya que, si la arquitectura ya soluciona sus problemas en su escala, la primera pregunta lógica es qué problemas no soluciona la arquitectura y que tentativamente podría solucionar el mundo molecular, o en la otra cara, partiendo de la conciencia del mundo molecular, que problemas nuevos podríamos identificar en la arquitectura.

Es decir, el mundo molecular nos abre la puerta a otra vista de la realidad, desde la cual podemos reanalizar la arquitectura, con la intención que en este ejercicio se pueda generar una imagen más nítida de la realidad arquitectónica.

En las últimas décadas y en términos de tecnología, la arquitectura vivió la llegada de las tecnologías digitales, los arquitectos nos encontramos con los 0 y 1 , hoy después ya de algunas décadas de las tecnólogas digitales, podemos ver, que la era digital transformó la manera de hacer arquitectura, con lo que hoy sería legítimo preguntarnos qué tanto nos cambiaran las tecnologías biológicas.

Por otro lado, los proyectos analizados demuestran, que algunas cualidades del espacio habitable pueden ser mejoradas desde el mundo molecular, como el aire, generando energía limpia o generando materiales con calidades programadas, estas referencias nos permiten teorizar en la posibilidad de que, en el futuro, o en un presente muy próximo, los arquitectos, diseñadores y urbanistas, resuelvan problemas de diseño programando microorganismos.

En este mismo discurso, sería prudente que algunos de los errores de la inclusión de las tecnologías digitales en la arquitectura, es que en algunos casos distrajo a los arquitectos y diseñadores de la función principal del diseño, aquí sería conveniente preguntarnos dos cosas, la primera cómo hacer para que las tecnologías biológicas no nos distraigan, y la segunda si tenemos que replantear la razón fundamental de la arquitectura y el diseño.

\section{Conclusiones}

Podemos concluir entonces, que, según los expuesto en el presente artículo, si es viable la inclusión de las tecnologías de la biología sintética en el diseño, actuando desde la manipulación directa del DNA, como con la generación de proteínas, con el metabolismo y con las relaciones entre microorganismos, así como con la programación de 
microrganismos de manera sintética y con la conciencia que el mundo molecular, no es la totalidad de la realidad, pero que si es una nueva perspectiva, de la cual, podemos extraer más detalles y nuevas visiones del espacio habitable, así como de los problemas y la manera de abordarlos.

Hoy deberíamos pensar cómo se relacionan los mundos de lo molecular y del espacio habitable, dejando como reto para los diseñadores, que no vean al mundo de la microbiología como el terreno de los microbiólogos, y dejando a las nuevas tecnologías bilógicas solamente como una herramienta sobre la mesa, el presente análisis, propone verlo como una nueva cara de la realidad, y en este sentido, de igual manera que los arquitectos y diseñadores incluyeron las tecnologías digitales en su práctica, pero también en su discurso, así las tecnologías biológicas podrían cambiar la forma en que hacemos arquitectura.

En términos efectivos, y como decíamos a mas cien años de biología sintética a diecisiete de la primera aplicación en la arquitectura, hoy tenemos la información e infraestructura tecnológica como para pensar en solucionar problemas concretos y tangibles desde el mundo molecular, en este artículo se expusieron varios proyectos y de todos ellos se mostró la secuencia genética, y al mismo tiempo hoy podríamos tenemos acceso a los materiales biológicos básicos para el desarrollo de seres sintéticos.

Con esto parece que el futuro ya llegó, parece que la pregunta efectiva es cuanto nos tardaremos los arquitectos $y$ diseñadores llegar. Pero el reto no se limita a aprender o en convertirnos en genetistas o en biólogos sintéticos, el verdadero reto, es qué hacemos con una tecnología tan potente en un momento donde la arquitectura tiene, al mismo tiempo, otros retos, como la emergencia social y la incapacidad que aún tenemos para hacer ciudades.

Dicho lo anterior, el reto es poner en la mesa los retos actuales, las oportunidades tecnológicas, y a partir de esto, generar respuestas que paso a paso nos aproximen al futuro de una arquitectura que pone los retos como oportunidades para ampliar la conciencia del espacio que habitamos.

\section{Agradecimientos}

Un reconocimiento especial para Alberto T. Estévez, Ernesto Ladrón de Guevara, Jorge A. Marcos Víquez, Guillermo I. López Domínguez, Andrea Llanes Figueroa y Salvador Amaro Rosas al FabLab Monterrey, Biohackademy, a la Maestría de Arquitecturas Avanzadas, al Centro Roberto Garza Sada de Arte, Arquitectura y Diseño, a la Vicerrectoría de Ciencias de la Salud de la Universidad de Monterrey, y a todos los que hicieron posible este proyecto.

\section{Referencias}

Aklujkar, M., Krushkal, J., DiBartolo, G., Lapidus, A., Land, M. L., \& Lovley, D. R. (2009). The genome sequence of Geobacter metallireducens: features of metabolism, physiology and regulation common and dissimilar to Geobacter sulfurreducens. BMC Microbiology, 9(1), 109. https://doi.org/10.1186/1471-21809-109
BioBricks Foundation | Biotechnology in the public interest. (2017). [BioBricks]. Retrieved June 25, 2017, from https://biobricks.org/

Estévez, A. T. (Ed.). (2004). Genetic Architectures / Arquitecturas genéticas (Bilingual edition). Barcelona: SITES.

Estévez, A. T. (2007). Genetic Barcelona Project. Retrieved June 26, 2017,

from http://www.albertoestevez.com/writing/escritos_geneticos/escrito s_geneticos08.pdf

Estévez, A. T. (2017). Genetic Architectures Research Group. Retrieved June 26, 2017, from http://geneticarchitectures.weebly.com/research_group.html

Geobacter Project. (2017). Retrieved June 26, 2017, from http://www.geobacter.org/about

GFP | BioBricks Foundation. (2017). Retrieved June 27, 2017, from https://biobricks.org/bpa/contributions/60/

Huang, Y.-H., Wu, H.-Y., Wu, K.-M., Liu, T.-T., Liou, R.-F., Tsai, S.-F., ... Yang, U.-C. (2013). Generation and Analysis of the Expressed Sequence Tags from the Mycelium of Ganoderma lucidum. PLoS ONE, 8(5), e61127. https://doi.org/10.1371/journal.pone.0061127

iGEM: International Genetically Engineered Machine. (2017). Retrieved June 25, 2017, from http://igem.org/Main_Page

Jansen, R., Embden, J. D. A. van, Gaastra, W., \& Schouls, L. M. (2002). Identification of genes that are associated with DNA repeats in prokaryotes. Molecular Microbiology, 43(6), 15651575.

Kumar, A. S., Lakshmanan, V., Caplan, J. L., Powell, D., Czymmek, K. J., Levia, D. F., \& Bais, H. P. (2012). Rhizobacteria Bacillus subtilis restricts foliar pathogen entry through stomata: Rhizobacteria and foliar infection. The Plant Journal, 72(4), 694706. https://doi.org/10.1111/j.1365-313X.2012.05116.x

Le Duc, S. (1910). Théorie physico-chimique de la vie et générations spontanées. Paris: A. Poinat.

Leibniz, G. W. (1981). Monadología. Oviedo: Grupo Helicón.

Meijer, W. J. (2014). Bacillus subtilis plasmid pTA1060, complete sequence. Retrieved from http://www.ncbi.nlm.nih.gov/nuccore/NC_001766.1

Mites: USDA ARS. (2017). Retrieved June 28, 2017, from https://www.ars.usda.gov/oc/kids/suburb/story2/mites/

Model, M. L. (2009). Bioinformatics Programming Using Python: Practical Programming for Biological Data. O'Reilly Media, Inc.

MycoWorks: Redefining Leather. (2017). Retrieved June 25, 2017, from http://www.mycoworks.com/

National Center for Biotechnology Information. (2017). Retrieved June 28, 2017, from https://www.ncbi.nlm.nih.gov/

Pena, A. (1988). Bioquímica. Editorial Limusa.

PhD, N. K., Bernstein, R., Ingram, K., \& Hart, K. M. (2015). BioBuilder: Synthetic Biology in the Lab. O'Reilly Media, Inc.

Rosenberg, L. E., \& Rosenberg, D. D. (2012). Human Genes and Genomes: Science, Health, Society. Academic Press.

Tortora, G. J., Funke, B. R., \& Case, C. L. (2007). Introducción a la microbiología. Ed. Médica Panamericana. 